\title{
Polish growth charts for preterm infants - comparison with reference Fenton charts
}

\author{
Izabela Mikulak, Maria Borszewska-Kornacka, \\ Joanna Puskarz-Gasowska, Renata Bokiniec(D) \\ Medical University of Warsaw, Poland
}

\begin{abstract}
Objectives: Proper infant classification, particularly a preterm infant, as small or large for gestational age, is crucial to undertake activities to improve postnatal outcomes.

This study aimed to assess the usability of the Fenton preterm growth charts to evaluate the anthropometric parameters of Polish preterm neonates.

Material and methods: In this single-center, retrospective study data extracted from the medical documentation of preterm neonates born 2002-2013 were analyzed. Body weight, body length, and head circumference were evaluated and used to develop growth charts, which were compared with the reference Fenton growth charts.

Results: This study included 3,205 preterm neonates, of whom 937 were born before 30 weeks of pregnancy. Overall, 11.04\%, $3.3 \%$, and $5.2 \%$ of neonates were below the $10^{\text {th }}$ percentile on the Fenton charts for birth weight, body length, and head circumference, respectively. Only 26 (6.67\%) of 390 analyzed anthropological parameters differed significantly between the study and the Fenton groups. Statistically significant differences between the study and the Fenton populations were found only in body length for both sexes, and in head circumference for female neonates.

Conclusions: The growth charts developed in this study for a population of Polish preterm neonates corresponded to the Fenton charts in terms of birth weight but differed in terms of body length and head circumference. Our findings suggest the need to evaluate growth charts for Polish preterm newborns.
\end{abstract}

Key words: growth charts; preterm neonate; intrauterine growth restriction; growth standards; Fenton

Ginekologia Polska 2021; 92, 12: 865-871

\section{INTRODUCTION}

Globally, approximately 15 million infants are born prematurely each year [1]. Complications associated with preterm birth are the leading cause of death in children under five years of age, accounting for more than one-third of deaths among neonates [2]. In Europe in 2014, 8.7\% of all births were preterm [3]. In Poland, the preterm birth rate has remained stable since 1996, and preterm neonates constitute approximately $6-7 \%$ of all neonates [3], corresponding to 30,000 births annually.

Birth weight is a key contributor to newborn mortality and developmental alterations. Birth weight is the primary indicator used in epidemiological reports; however, it only poorly characterizes newborn maturity. Nevertheless, it reflects the role of genetic and nutritional factors and allows dystrophic neonates to be distinguished independently of their gestational age.
For neonate assessment, growth charts are used to evaluate birth weight, length, and head, abdominal, and chest circumference [4]. Yudkin et al. [5], developed fetal growth charts for the British population in 1987, and more recently Dubiel et al. [6], developed growth charts for the Polish population. The Polish Neonatal Society recommends application of the Fenton's growth charts to evaluate preterm newborns with a gestational age of 23-32 weeks [7]. These charts were initially developed by Fenton et al. [8] in 2003, based on systematic review and meta-analysis of published reference studies. In 2013, Fenton et al. [7], published a revised version of these charts, resulting from a large meta-analysis including nearly 4 million preterm births in Germany, the USA, Italy, Australia, Scotland, and Canada. Recently, Kajdy et al. [9], developed growth charts for a population of nearly 38,000 Polish singleton neonates, excluding those with major congenital defects and infections. 


\section{Objectives}

Proper infant classification, particularly a preterm infant, as small or large for gestational age, is crucial to undertake activities to improve postnatal outcomes. This study aimed to assess the usability of the Fenton preterm growth charts to evaluate Polish preterm newborns, which is in line with the current Polish Neonatal Society recommendations. To do so, we developed for the first-time growth charts for Polish preterm neonates (from the Masovian region) and compared them with the Fenton growth charts.

\section{MATERIAL AND METHODS}

This retrospective study was based on data extracted from the medical documentation of pregnant women and neonates born at the Second Department of Obstetrics and Gynecology, Medical University of Warsaw, Poland, and hospitalized at the Neonatal and Intensive Care Department, Medical University of Warsaw, between 1.01.2002 and 31.12.2013. The study was approved by the Bioethics Committee of the Medical University of Warsaw. All neonates born before 37 weeks of gestation were considered preterm and included in the study. Gestational age was determined based on the date of the first day of the last menstrual period, recorded in the hospital medical documentation or maternity notes. Moreover, a first-trimester ultrasound, during which gestational age was determined by fetal biometry, was routinely performed at our center.

Body weight, length, and head circumference were evaluated and used to develop the growth charts. For some neonates, particularly those who were extremely immature, only weight and length measurements were conducted due to their poor condition at birth.

Birth weight measurements were performed using dedicated WPT 6/15D neonatal scales (Radwag, Poland) located in delivery and operating rooms. Length and head circumference measurements were performed with a tape measure. The crown-heel length (the distance from the crown of the head to the heel, including natural flexures caused by physiological muscle tension) was expressed in centimeters $(\mathrm{cm})$. For head circumference, the occipital frontal circumference (measured above the ears and approximately $1 \mathrm{~cm}$ above the eyebrows) was used.

Statistical analyses were performed using SAS v. 14.1 (SAS Institute, NC, USA). The anthropometric parameters were used to build a quantile regression model, which allowed percentile curves to be evaluated and hypotheses regarding differences between the developed and the reference growth charts to be tested. $P$ values less than 0.05 were considered significant.

\section{RESULTS \\ Study group}

A total of 3,205 preterm neonates ( $45.5 \%$ female) were included in the study, of whom 937 were born before 30 weeks of pregnancy. The study group was divided into 15 subgroups, depending on maturity (i.e., gestational age): neonates born with a gestational age of 22 weeks $(n=26)$, 23 weeks $(n=69), 24$ weeks $(n=93), 25$ weeks $(n=111)$, 26 weeks $(n=142), 27$ weeks $(n=134), 28$ weeks $(n=184)$, 29 weeks $(n=178), 30$ weeks $(n=237), 31$ weeks $(n=153)$, 32 weeks $(n=211), 33$ weeks $(n=242), 34$ weeks $(n=337)$, 35 weeks $(n=446)$, and 36 weeks $(n=642)$. The study included 1,853 (66.69\%) infants from singleton, 817 (29.39\%) from twin, 102 (3.67\%) from triplet, and 8 (0.28\%) from quadruplet gestations. In the study group, 374 mothers (16.27\%) were diagnosed with gestational diabetes mellitus (GDM), and 67 (2.92\%) with pre-gestational diabetes mellitus.

Overall, $11.04 \%$ of neonates had a birth weight below the $10^{\text {th }}$ percentile according to the Fenton charts and were classified as small for gestational age (SGA) (Tab. 1). The highest discrepancies between the charts regarding SGA classification were observed for neonates born with a gestational age of 28 weeks, of whom $25.2 \%$ were classified as SGA, and those with a gestational age of 36 weeks, of whom $7 \%$ were classified as SGA, according to the Fenton charts. Only $3.3 \%$ of preterm neonates had body length and $5.2 \%$ head circumference below the $10^{\text {th }}$ percentile on the Fenton charts at birth (Tab. 1).

\begin{tabular}{|c|c|c|c|}
\hline $\begin{array}{l}\text { Gestational } \\
\text { age, weeks }\end{array}$ & $\begin{array}{l}\text { Birth body } \\
\text { length }\end{array}$ & Birth weight & $\begin{array}{l}\text { Birth head } \\
\text { circumference }\end{array}$ \\
\hline 24 & $10.1 \%$ & $13.9 \%$ & $13.9 \%$ \\
\hline 25 & $4.4 \%$ & $11.0 \%$ & $3.3 \%$ \\
\hline 26 & $4.1 \%$ & $9.0 \%$ & $6.6 \%$ \\
\hline 27 & $1.7 \%$ & $14.3 \%$ & $3.4 \%$ \\
\hline 28 & $7.5 \%$ & $25.2 \%$ & $13.6 \%$ \\
\hline 29 & $3.4 \%$ & $15.5 \%$ & $6.1 \%$ \\
\hline 30 & $3.1 \%$ & $13.3 \%$ & $7.7 \%$ \\
\hline 31 & $1.4 \%$ & $15.6 \%$ & $2.8 \%$ \\
\hline 32 & $1.0 \%$ & $10.1 \%$ & $1.0 \%$ \\
\hline 33 & $2.6 \%$ & $11.9 \%$ & $4.4 \%$ \\
\hline 34 & $2.9 \%$ & $8.3 \%$ & $4.8 \%$ \\
\hline 35 & $2.3 \%$ & $7.8 \%$ & $3.4 \%$ \\
\hline 36 & $3.9 \%$ & $7.0 \%$ & $5.2 \%$ \\
\hline Overall & $3.3 \%$ & $11.04 \%$ & $5.2 \%$ \\
\hline
\end{tabular}




\section{Body length}

Female neonates from the study group had greater birth body length than those from the Fenton population at each percentile and gestational age (Fig. 1A). Statistically significant differences between the study and the Fenton group were observed for the $50^{\text {th }}$ percentile at gestational ages of 31 weeks $(p=0.045), 32$ weeks $(p=0.037), 34$ weeks $(p=0.033), 35$ weeks $(p=0.024)$, and 36 weeks $(p=0.022)$, and for the $90^{\text {th }}$ percentile at gestational ages of 28 weeks $(p=0.047), 32$ weeks $(p=0.047), 33$ weeks $(p=0.044)$, 34 weeks ( $p=0.037), 35$ weeks $(p=0.048)$, and 36 weeks $(p=0.045)$.

Male neonates in this study group had greater birth body length than those from the Fenton population at most gestational ages and percentiles (Fig. 2A). Nine percent of neonates in the study group had a smaller body length than the reference group. Statistically significant differences between the study and the Fenton group were observed for the $50^{\text {th }}$ percentile at gestational ages of 28 weeks $(p=0.046), 30$ weeks $(p=0.048), 31$ weeks $(p=0.048)$, 33 weeks $(p=0.048)$, and 36 weeks $(p=0.030)$, and for the $90^{\text {th }}$ percentile at gestational ages of 27 weeks $(p=0.0498)$, 33 weeks $(p=0.049)$, and 36 weeks $(p=0.049)$.

\section{Birth weight}

Both female and male preterm neonates from the study group had greater birth weight than those from the Fenton population at most gestational ages and percentiles (Fig. 1B and $2 \mathrm{~B}$ for female and male neonates, respectively). Twenty-three percent of female neonates and $17 \%$ of male neonates in the study group had a smaller birth weight than the reference group. No statistically significant differences in birth weight were observed between the groups.

\section{Head circumference}

Both female and male preterm neonates from the study group had larger birth head circumferences than those from the Fenton population at most gestational ages and percentiles (Fig. 1C and $2 \mathrm{C}$ for female and male neonates, respectively). Nine percent of female and $12 \%$ of male neonates in the study group had smaller head circumference than the reference group. In female infants, statistically significant differences between the study and the Fenton groups were observed for the $50^{\text {th }}$ percentile at gestational ages of 24 weeks $(p=0.026), 25$ weeks $(p=0.015), 26$ weeks ( $p=0.009), 27$ weeks $(p=0.011), 28$ weeks $(p=0.020)$, 29 weeks $(p=0.032)$, and 30 weeks $(p=0.042)$. In male neonates, no statistically significant differences in head circumference were observed between the groups.
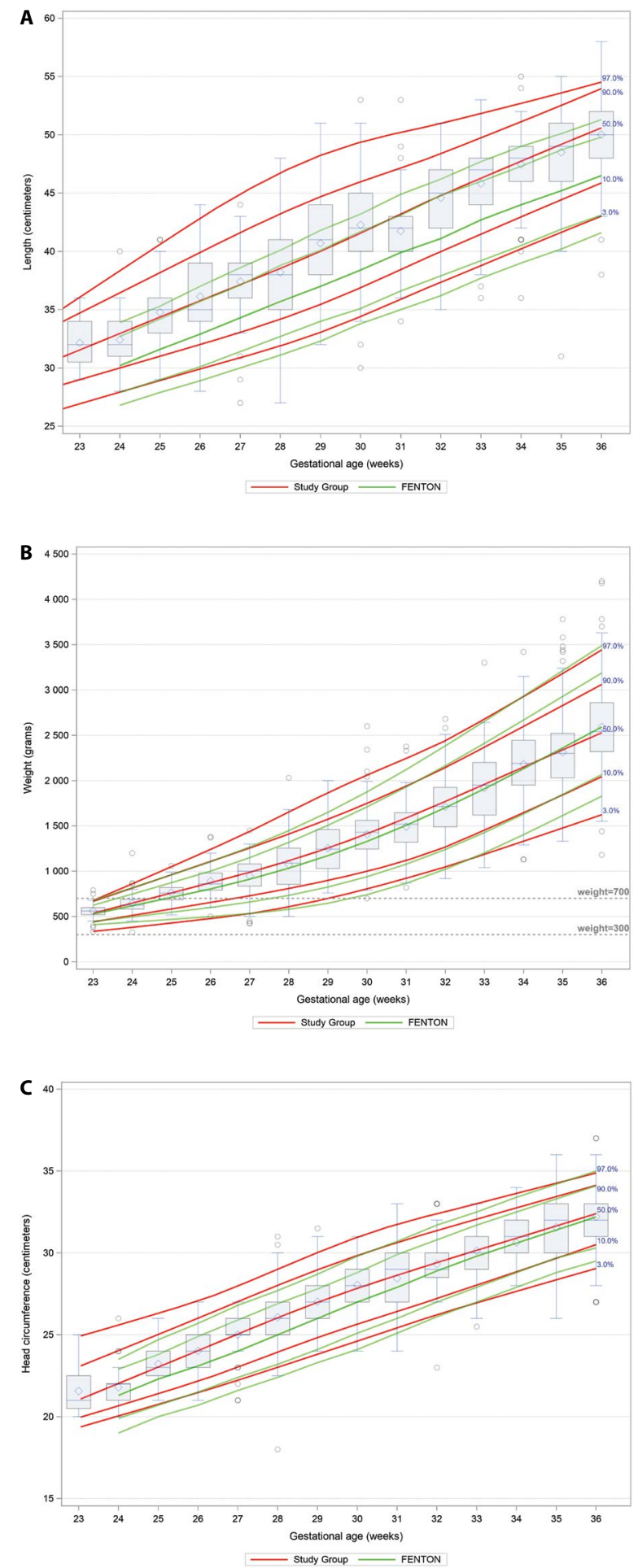

Figure 1. Growth charts for female Polish preterm neonates (red lines) compared with the Fenton reference charts (green lines); A. Body length; B. Birth weight; C. Head circumference. For each subgroup of neonates, boxes represent interquartile ranges, horizontal lines represent median values, diamonds represent mean values, whiskers represent minimum and maximum values, and circles correspond to outliers 

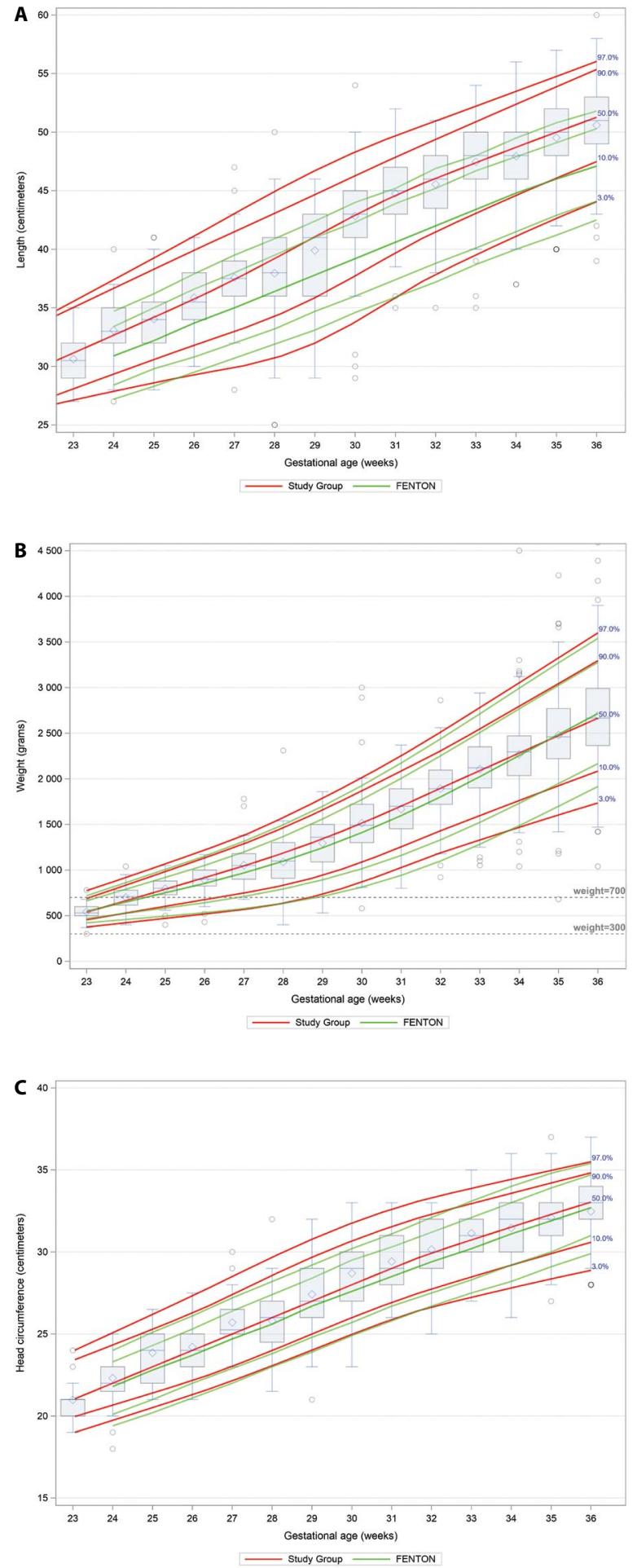

Figure 2. Growth charts for male Polish preterm neonates (red lines) compared with the Fenton reference charts (green lines); A. Body length; B. Birth weight; C. Head circumference. For each subgroup of neonates, boxes represent interquartile ranges, horizontal lines represent median values, diamonds represent mean values, whiskers represent minimum and maximum values, and circles correspond to outliers

\section{Overall analysis}

Only 26 (6.67\%) of 390 analyzed anthropological parameters differed significantly between the study group and the Fenton group. Statistically significant differences were observed in appropriate for gestational age (AGA, $3.77 \%$ ) and large for gestational age (LGA, 2\%) neonates, and mostly involved body length and head circumference. No significant differences were reported for SGA neonates.

\section{DISCUSSION}

In this study, we developed growth charts for Polish preterm neonates based on anthropological parameters reported for 3,205 newborn babies delivered between 2002 and 2013. We showed that Polish preterm infants display certain discrepancies in body length and head circumference compared with the Fenton population, but significant differences were observed in only $6.67 \%$ of 390 analyzed anthropological parameters.

Morphologically and physiologically immature preterm neonates are susceptible to severe and long-term complications, especially if they are affected by growth alterations. Therefore, classification of a preterm neonate as SGA or LGA determines the activities to be conducted by the healthcare professionals and enables the improved management of potential threats. SGA infants, particularly preterm neonates, have an increased risk of neonatal and post-neonatal mortality $[10,11]$.

The first broadly used growth charts were developed by Lubchenco et al. [12], in 1966, and were based on 4,700 neonates born between the $26^{\text {th }}$ and $42^{\text {nd }}$ week of gestation. The terms AGA, SGA, and LGA to categorize neonatal nutritional state were introduced by Battaglia and Lubchenco in 1967 [13]. Babson and Benda's [14] intrauterine growth charts from 1976, developed for Caucasian neonates born between the $27^{\text {th }}$ and $44^{\text {th }}$ week of gestation, were updated in 2003 by Fenton [8]. In Poland, the Fenton growth charts in their revised version from 2013 [7] are currently recommended, and are routinely used for the growth evaluation of preterm infants born between the $23^{\text {rd }}$ and $32^{\text {nd }}$ week of pregnancy. For infants over 50 weeks of age, the WHO growth charts [15], or the Polish growth charts, particularly those developed by The Children's Memorial Health Institute, are used.

In our study, $11.04 \%, 3.3 \%$, and $5.2 \%$ of preterm neonates were below the $10^{\text {th }}$ percentile on the Fenton charts for birth weight, body length, and head circumference, respectively. Similar to our findings, Tuzun et al. [16], showed that $12 \%$ of preterm neonates were classified as SGA according to the Fenton charts. By contrast, a corresponding study 
of 603 preterm neonates in India identified 16.1\%, 14.4\%, and $11.3 \%$ infants as having intrauterine growth restriction (IUGR; i.e., below the $10^{\text {th }}$ percentile) according to the Fenton charts for birth weight, body length, and head circumference, respectively [17]. This indicates that the percentage of preterm neonates who are classified as SGA may differ depending on the characteristics of the population evaluated. A recent study by Kajdy et al. [9], classified $9.8 \%$ of singleton neonates born between weeks 23 and 40 of pregnancy as SGA, and $10.27 \%$ as LGA, but only $5.01 \%$ of SGA neonates were identified with the Fenton charts. This percentage was higher when exclusively preterm neonates were evaluated. The lower percentage of SGA infants in the population evaluated by Kajdy et al. [9], than in our population may be caused by the different inclusion criteria applied. Our study group consisted of consecutive neonates, including those from multifetal pregnancies, as well as those with congenital defects and infections or karyotype abnormalities, and therefore may better represent the general newborn population described by the Fenton growth charts than the population studied by Kajdy et al. [9],

In 2015, The International Fetal and Newborn Growth Consortium for the 21st Century (INTERGROWTH-21st) Study Group developed a new newborn weight standard [18]. Aimed at better discrimination of SGA infants, INTERGROWTH-21st is based on birth weights observed among pregnancies characterized by optimal fetal growth and good maternal health status, from diverse geographical regions. INTERGROWTH-21st charts tend to identify significantly more SGA infants at birth than the Fenton charts describing the general population $[16,17]$. In a study by Reddy et al. [17], infants classified as SGA by INTERGROWTH-21st charts and not by Fenton charts were at higher risk of morbidities. However, such a relationship was not reported by Tuzun et al. [16].

An alternative approach to infant classification is the customized gestation-related optimal weight (GROW) standard. The GROW standard, using the most widely implemented model (developed by Gardosi et al.) [19-21], takes into account maternal height and weight, parity, ethnic origin, and gestational age at delivery, and thus estimates an individualized, ideal neonate birth weight for a particular birth. Recent studies evaluated the usefulness of INTERGROWTH-21st in a general obstetrics population and collated it with customized GROW centiles and/or population standards. Compared with INTERGROWTH-21st, customized standards identified more SGA infants at risk of mortality and morbidity in populations in New Zealand [22], Australia [23], Sweden [24], and in a multinational analysis from 10 countries [25].

Both population birth weight standards and sonographic fetal growth charts can be used to identify preterm neonates who are SGA. Preterm infants have lower birth weights than would be expected by estimating the weight of fetuses that remain in utero to term [26]. Therefore, fetal growth standards usually classify a higher percentage of preterm infants as SGA than birth weight charts. In 2019, Pritchard et al. [27], compared the performance of five growth charts in the identification of infants at a high risk of adverse perinatal outcomes. Intrauterine charts identified a significantly higher proportion of SGA infants in the preterm population than birth weight charts [27]. Among fetal growth charts, INTERGROWTH-21st identified the smallest percentage of infants as SGA. This subpopulation was at the highest risk of mortality and morbidity. GROW charts were the most accurate at detecting individuals with an increased risk of adverse outcomes [27]. Using population birth weight charts, $10.7 \%$ of preterm infants were classified as below the $10^{\text {th }}$ percentile, and $5.6 \%$ below the $5^{\text {th }}$ percentile [27]. Similar percentages were observed in our study: according to the Fenton charts, $11.04 \%$ of preterm neonates were born with a weight below the $10^{\text {th }}$ percentile, and $5.28 \%$ below the $5^{\text {th }}$ percentile. Large discrepancies in SGA estimates conducted with various growth charts were observed by Kajdy et al. [9]. In a population of Polish neonates, 5.01\% of newborns were identified as SGA according to the Fenton charts, 3.88\% according to the Dubiel [6] percentiles, and only $2.33 \%$ according to INTERGROWTH-21st [9]. These findings might justify the adjustment of global charts to account for the characteristics of the given population [9]. Indeed, the most accurate estimation of the prevalence of SGA infants in the Polish population (10.12\%) [9] was made with charts developed by Mikolajczyk et al., [28], which include the Gardosi customization [19], therefore allow percentiles to be adjusted to the local population.

In a study from 2018, Landau-Crangle et al. [29], compared different approaches to predict individualized postnatal growth trajectories in preterm infants based on the WHO Fenton charts. Incorporation of the growth velocity approach, which accounts for the recently described physiological postnatal adaptation period in preterm infants, enabled growth trajectories to accurately meet WHO target weights at a post-menstrual age of 42 weeks [29]. The use of adequate tools to monitor appropriate postnatal growth could enable the improved classification of extrauterine growth restriction (EUGR) [29], currently defined as a weight trajectory evolving below the $10^{\text {th }}$ percentile on intrauterine growth charts [30]. A similar recommendation was recently made for IUGR, which suggested that not only infants with a birth weight below the $10^{\text {th }}$ percentile, but also those with a trend of falling percentiles, should be classified as having IUGR [31]. Indeed, not only SGA infants, but also those with IUGR or/and EUGR, have a likely prognosis of inappropriate body composition and impaired neurodevelopment. 
We reported statistically significant differences between the charts developed within this study and the Fenton charts, mainly for AGA and LGA neonates in terms of body length and head circumference. In our population, $11.04 \%$ of neonates were classified as SGA and $6.44 \%$ as LGA according to the Fenton charts for birth weight. A higher proportion of LGA can be explained, at least in part, by the high percentage of newborns delivered by mothers diagnosed with diabetes mellitus in our study. GDM affects approximately $7 \%$ of all pregnancies worldwide [32]. In Europe, an overall GDM prevalence of 5.4\% (3.8-7.8\%) was reported [33]. In Poland, GDM is diagnosed in $0.7-12.3 \%$ of pregnancies [34]. The higher percentage of patients with GDM in our study (16.27\%) is a result of the characteristics of our center, which is oriented to manage pregnancies complicated by GDM, among other complications. High proportion of preterm neonates with SGA born in 28th week may be associated with the referral level of the center, which provides high-risk pregnancy care.

The main limitation of this study is its retrospective nature. Additionally, data were collected from a single, highly specialized site; therefore, although the study group consists of consecutive preterm neonates, it may not be representative of the whole Polish population.

\section{CONCLUSIONS}

To conclude, we developed growth charts for preterm neonates born in Poland, corresponding to the Fenton growth charts. Reported here statistically significant differences in body length and head circumference between the charts suggest the need to evaluate growth charts specifically for Polish preterm newborns. To ensure a representative profile of Polish preterm infants, this should be done with a larger cohort of patients and with a multicenter approach. Moreover, growth trajectories during prenatal and postnatal periods would be worth evaluating, as they could predict possible adverse outcomes.

\section{Acknowledgments}

The authors would like to thank Proper Medical Writing Sp. $z$ o.o. for providing medical writing assistance and editorial support in the development of this manuscript.

\section{Conflict of interests}

The authors report no conflict of interest.

\section{REFERENCES}

1. Chawanpaiboon S, Vogel JP, Moller AB, et al. Global, regional, and national estimates of levels of preterm birth in 2014: a systematic review and modelling analysis. Lancet Glob Health. 2019; 7(1): e37-e46, doi: 10.1016/S2214-109X(18)30451-0, indexed in Pubmed: 30389451.

2. UN Inter-Agency Group for Child Mortality. Estimation Levels and trends in child mortality: Report 2017. New York: United Nations Children's
Fund. https://www.unicef.org/publications/files/Child_Mortality_Report_2017.pdf (2020-02-25).

3. Zeitlin J, Szamotulska K, Drewniak N, et al. Euro-Peristat Preterm Study Group. Preterm birth time trends in Europe: a study of 19 countries. BJOG. 2013; 120(11): 1356-1365, doi: 10.1111/1471-0528.12281, indexed in Pubmed: 23700966.

4. Shrestha S, Thakur A, Goyal S, et al. Growth charts in neonates. Curr Med Res Pract. 2016; 6(2): 79-84, doi: 10.1016/j.cmrp.2016.03.009.

5. Yudkin PL, Aboualfa M, Eyre JA, et al. New birthweight and head circumference centiles for gestational ages 24 to 42 weeks. Early Hum Dev. 1987; 15(1): 45-52, doi: 10.1016/0378-3782(87)90099-5, indexed in Pubmed: 3816638.

6. Dubiel M, Krajewski M, Pietryga M, et al. Fetal biometry between 20-42 weeks of gestation for Polish population. Ginekol Pol. 2008; 79(11): 746-753, indexed in Pubmed: 19140496.

7. Fenton TR, Kim JH. A systematic review and meta-analysis to revise the Fenton growth chart for preterm infants. BMC Pediatr. 2013; 13: 59, doi: 10.1186/1471-2431-13-59, indexed in Pubmed: 23601190.

8. Fenton TR. A new growth chart for preterm babies: Babson and Benda's chart updated with recent data and a new format. BMC Pediatr. 2003; 3: 13, doi: 10.1186/1471-2431-3-13, indexed in Pubmed: 14678563.

9. Kajdy A, Modzelewski J, Filipecka-Tyczka D, et al. Development of birth weight for gestational age charts and comparison with currently used charts: defining growth in the Polish population. J Matern Fetal Neonatal Med. 2019 [Epub ahead of print]: 1-8, doi: 10.1080/14767058.2019.1676412, indexed in Pubmed: 31619090.

10. Katz J, Wu LA, Mullany LC, et al. Prevalence of small-for-gestational-age and its mortality risk varies by choice of birth-weight-for-gestation reference population. PLoS One. 2014; 9(3): e92074, doi: 10.1371/journal. pone.0092074, indexed in Pubmed: 24642757.

11. Katz J, Lee ACc, Kozuki N, et al. CHERG Small-for-Gestational-Age-Preterm Birth Working Group. Mortality risk in preterm and small-for-gestational-age infants in low-income and middle-income countries: a pooled country analysis. Lancet. 2013; 382(9890): 417-425, doi: 10.1016/S01406736(13)60993-9, indexed in Pubmed: 23746775.

12. Lubchenco LO, Hansman C, Boyd E. Intrauterine growth in length and head circumference as estimated from live births at gestational ages from 26 to 42 weeks. Pediatrics. 1966; 37(3): 403-408, indexed in Pubmed: 5906365.

13. Battaglia FC, Lubchenco LO. A practical classification of newborn infants by weight and gestational age. J Pediatr. 1967; 71(2): 159-163, doi: 10.1016/s0022-3476(67)80066-0, indexed in Pubmed: 6029463.

14. Babson SG, Benda GI. Growth graphs for the clinical assessment of infants of varying gestational age. J Pediatr. 1976; 89(5): 814-820, doi: 10.1016/s0022-3476(76)80815-3, indexed in Pubmed: 978333.

15. WHO Multicentre Growth Reference Study Group. WHO Child Growth Standards based on length/height, weight and age. Acta Paediatr Suppl. 2006; 450: 76-85, doi: 10.1111/j.1651-2227.2006.tb02378.x, indexed in Pubmed: 16817681

16. Tuzun F, Yucesoy E, Baysal B, et al. Comparison of INTERGROWTH-21 and Fenton growth standards to assess size at birth and extrauterine growth in very preterm infants. J Matern Fetal Neonatal Med. 2018; 31(17): 2252-2257, doi: 10.1080/14767058.2017.1339270, indexed in Pubmed: 28587527.

17. Reddy KV, Sharma D, Vardhelli V, et al. Comparison of Fenton 2013 growth curves and Intergrowth-21 growth standards to assess the incidence of intrauterine growth restriction and extrauterine growth restriction in preterm neonates $\leq 32$ weeks. J Matern Fetal Neonatal Med. 2019 [Epub ahead of print]: 1-8, doi: 10.1080/14767058.2019.1670795, indexed in Pubmed: 31656092.

18. Kozuki N, Katz J, Christian P, et al. Child Health Epidemiology Reference Group Preterm Birth-SGA Working Group. Comparison of US birth weight references and the international fetal and newborn growth consortium for the 21st century standard. JAMA Pediatr. 2015; 169(7): e151438, doi: 10.1001/jamapediatrics.2015.1438, indexed in Pubmed: 26147059.

19. Gardosi J, Chang A, Kalyan B, et al. Customised antenatal growth charts. Lancet. 1992; 339(8788): 283-287, doi: 10.1016/01406736(92)91342-6, indexed in Pubmed: 1346292.

20. Gardosi J, Mongelli $M$, Wilcox $M$, et al. An adjustable fetal weight standard. Ultrasound Obstet Gynecol. 1995; 6(3): 168-174, doi: 10.1046/j.146 9-0705.1995.06030168.x, indexed in Pubmed: 8521065.

21. Gardosi J. Customized fetal growth standards: rationale and clinical application. Seminars in Perinatology. 2004; 28(1): 33-40, doi: 10.1053/j. semperi.2003.12.002. 
22. Anderson NH, Sadler LC, McKinlay CJD, et al. INTERGROWTH-21st vs customized birthweight standards for identification of perinatal mortality and morbidity. Am J Obstet Gynecol. 2016; 214(4): 509.e1-509.e7, doi: 10.1016/j.ajog.2015.10.931, indexed in Pubmed: 26546850.

23. Pritchard N, Lindquist A, Siqueira ID, et al. INTERGROWTH-21 st compared with GROW customized centiles in the detection of adverse perinatal outcomes at term. J Matern Fetal Neonatal Med. 2020; 33(6): 961-966, doi: 10.1080/14767058.2018.1511696, indexed in Pubmed: 30372647.

24. Vieira MC, Relph S, Persson $M$, et al. Determination of birth-weight centile thresholds associated with adverse perinatal outcomes using population, customised, and Intergrowth charts: A Swedish population-based cohort study. PLoS Med. 2019; 16(9): e1002902, doi: 10.1371/journal. pmed.1002902, indexed in Pubmed: 31539391.

25. Francis A, Hugh O, Gardosi J. Customized vs INTERGROWTH-21 standards for the assessment of birthweight and stillbirth risk at term. Am J Obstet Gynecol. 2018; 218(2S): S692-S699, doi: 10.1016/j.ajog.2017.12.013, indexed in Pubmed: 29422208.

26. Burkhardt T, Schäffer L, Zimmermann R, et al. Newborn weight charts underestimate the incidence of low birthweight in preterm infants. Am J Obstet Gynecol. 2008; 199(2): 139.e1-139.e6, doi: 10.1016/j. ajog.2008.01.011, indexed in Pubmed: 18395687.

27. Pritchard NL, Hiscock RJ, Lockie E, et al. Identification of the optimal growth charts for use in a preterm population: An Australian state-wide retrospective cohort study. PLoS Med. 2019; 16(10): e1002923, doi: 10.1371/journal.pmed.1002923, indexed in Pubmed: 31584941.
28. Mikolajczyk RT, Zhang J, Betran AP, et al. A global reference for fetal-weight and birthweight percentiles. Lancet. 2011; 377(9780): 1855-1861, doi: 10.1016/S0140-6736(11)60364-4, indexed in Pubmed: 21621717.

29. Landau-Crangle E, Rochow N, Fenton TR, et al. Individualized postnatal growth trajectories for preterm infants. JPEN J Parenter Enteral Nutr. 2018; 42(6): 1084-1092, doi: 10.1002/jpen.1138, indexed in Pubmed: 29419902.

30. Clark RH, Thomas $P$, Peabody J. Extrauterine growth restriction remains a serious problem in prematurely born neonates. Pediatrics. 2003; $111(5 \mathrm{Pt}$ 1): $986-990$, doi: $10.1542 /$ peds.111.5.986, indexed in Pubmed: 12728076.

31. Royal College of Obstetricians and Gynaecologists. The investigation and management of the small-for-gestational-age fetus. RCOG Green-top Guideline No. 31, 2 edition; 2013. https://www.rcog.org.uk/globalassets/documents/guidelines/gtg_31.pdf (2020-02-25).

32. American Diabetes Association. Position statement: gestational diabetes mellitus. Diabetes Care. 2003; 26(Suppl 1): S103-S105, doi: 10.2337/diacare.26.2007.s103, indexed in Pubmed: 12502631.

33. Eades CE, Cameron DM, Evans JMM. Prevalence of gestational diabetes mellitus in Europe: A meta-analysis. Diabetes Res Clin Pract. 2017; 129: 173-181, doi: 10.1016/j.diabres.2017.03.030, indexed in Pubmed: 28531829.

34. Wilczyński J, Dziatosz K. Cukrzyca ciążowa - ryzyko dla matki i jej dziecka [Gestational diabetes mellitus — risks for mother and child]. Perinatol Neonatol i Ginekol. 2009; 2: 85-89. 